\title{
Quantum pumping and dissipation: from closed to open systems
}

\author{
Doron Cohen \\ Department of Physics, Ben-Gurion University, Beer-Sheva 84105, Israel
}

\begin{abstract}
Current can be pumped through a closed system by changing parameters (or fields) in time. The Kubo formula allows to distinguish between dissipative and non-dissipative contributions to the current. We obtain a Green function expression and an $S$ matrix formula for the associated terms in the generalized conductance matrix: the "geometric magnetism" term that corresponds to adiabatic transport; and the "Fermi golden rule" term which is responsible to the irreversible absorption of energy. We explain the subtle limit of an infinite system, and demonstrate the consistency with the formulas by Landauer and Büttiker, Prétre and Thomas. We also discuss the generalization of the fluctuation-dissipation relation, and the implications of the Onsager reciprocity.
\end{abstract}

Linear response theory (LRT) is the traditional theoretical tool for dealing with the response of driven systems 1, 2, 3, 4]. It offers an expression (the Kubo formula) for the generalized susceptibility, and hence for the generalized conductance matrix. It has been realized that in the adiabatic limit the Kubo formula reduces to an expression for "geometric magnetism" 5]. In case of electrical current calculation the latter gives the "adiabatic transport" of charge [6, 7]. Outside of the adiabatic regime the response includes an additional "dissipation" term 8]. The latter determines the rate of irreversible energy absorption, which is caused by Fermi-golden-rule transitions between energy levels.

Recently there was much interest in analyzing the response of open systems that are connected to reservoirs. The analysis has been based on the $S$-matrix formalism, leading to the Landauer formula [2, 3], and more generally to the Büttiker, Prétre and Thomas (BPT) formula [9]. A major motivation for the present work is the realization that the relation between the BPT formula and the Kubo formula has not been clarified. In particular the notion of "adiabatic pumping" in the context of an open system has been left obscured, and some confusion has arose regarding the role of dissipation in the pumping process [8, 10, 11].

The purpose of the present work is to analyze the response of closed isolated systems [11, 12], and in particular to consider the special limit of an infinite system (no reservoirs!). Thus we are going to construct a bridge between the LRT formulation and the BPT formula. This is of great practical importance, because the assumed open geometry of the $S$-matrix formulation is in many cases an idealization. It is clear that Kubo formula allows a straightforward incorporation of finite-size, external noise, environmental and possibly also many-body effects. A major step in constructing this bridge, had been taken in Ref. [13], where the authors start with the Kubo formula for the electrical conductivity and end up with the Landauer formula which relates the conductance to the transmission of the device. We are going to see that the general case, which deals with the generalized conductance matrix, and hence incorporates adiabatic transport, is much more subtle.

Consider a closed isolated system whose Hamiltonian $\mathcal{H}$ depends on several control parameters $x_{j}$. An example is presented in Fig. 1, where $x_{1}$ and $x_{2}$ are gate voltages and $x_{3}$ is the magnetic flux through the loop. The generalized forces are conventionally defined as $F^{k}=-\partial \mathcal{H} / \partial x_{k}$. Note that $F^{3}$ is the electrical current. In LRT [1 the first order contribution to $\left\langle F^{k}\right\rangle$ is related to $x_{j}(t)$ by a causal response kernel $\alpha^{k j}\left(t-t^{\prime}\right)$. The Kubo expression for this response kernel is $\alpha^{k j}(\tau)=\Theta(\tau) K^{k j}(\tau)$, where $K^{k j}(\tau)=(i / \hbar)\left\langle\left[F^{k}(\tau), F^{j}(0)\right]\right\rangle$, and $\Theta(\tau)$ is the step function. The Fourier transform of $\alpha^{k j}(\tau)$ is the generalized susceptibility $\chi^{k j}(\omega)$. The generalized conductance matrix is:

$$
\boldsymbol{G}^{k j}=\lim _{\omega \rightarrow 0} \frac{\operatorname{Im}\left[\chi^{k j}(\omega)\right]}{\omega}=\int_{0}^{\infty} K^{k j}(\tau) \tau d \tau
$$

Thus in the limit of zero frequency the non-trivial part of the response can be written as a generalized Ohm law

$$
\left\langle F^{k}\right\rangle=-\sum_{j} \boldsymbol{G}^{k j} \dot{x}_{j} \equiv(-\boldsymbol{\eta} \cdot \dot{x}-\boldsymbol{B} \wedge \dot{x})_{k}
$$

where following [5] the generalized conductance matrix is written as a sum of a symmetric matrix $\boldsymbol{\eta}^{k j}=\boldsymbol{\eta}^{i k}$ that represents the dissipative response, and an antisymmetric matrix $\boldsymbol{B}^{k j}=-\boldsymbol{B}^{j k}$ that represents the non-dissipative response (also called "geometric magnetism").

For a device as in Fig.1, and zero temperature occupation of non-interacting (spinless) Fermions, we find below that the dissipative part of the response is

$$
\begin{aligned}
\boldsymbol{\eta}^{k j} & =\frac{\hbar}{\pi} \operatorname{trace}\left[F^{k} \operatorname{Im}\left[\mathrm{G}^{+}\right] F^{j} \operatorname{Im}\left[\mathrm{G}^{+}\right]\right] \\
& =\frac{\hbar}{4 \pi} \operatorname{trace}\left[\frac{\partial S^{\dagger}}{\partial x_{i}} \frac{\partial S}{\partial x_{j}}\right]
\end{aligned}
$$

where $\mathrm{G}^{ \pm}=1 /(E-\mathcal{H} \pm i 0)$ are Green functions of the corresponding open system, and $\operatorname{Im}\left[\mathrm{G}^{+}\right]=-i \frac{1}{2}\left(\mathrm{G}^{+}-\mathrm{G}^{-}\right)$. For the non-dissipative part of the response we find:

$$
\begin{aligned}
\boldsymbol{B}^{k j} & =-\frac{i \hbar}{2 \pi} \operatorname{trace}\left[F^{k}\left(\mathrm{G}^{+}+\mathrm{G}^{-}\right) F^{j} \operatorname{Im}\left[\mathrm{G}^{+}\right]\right] \\
& =\frac{e}{4 \pi i} \operatorname{trace}\left[P_{\mathrm{A}}\left(\frac{\partial S}{\partial x_{j}} S^{\dagger}-\frac{\partial S^{\dagger}}{\partial x_{j}} S\right)\right]+\boldsymbol{B}_{\mathrm{intrf}}^{3 j}(6)
\end{aligned}
$$


where the second equality holds for $k=3$, and allows the determination of the electrical current $\left\langle F^{3}\right\rangle$ via a specified lead A. The last term is defined in Eq.(26). The projector $P_{\mathrm{A}}$ restricts the trace operation to be over the specified lead channels. In the absence of magnetic field the remaining component is $\boldsymbol{B}^{12}=0$, while $\boldsymbol{\eta}^{31}=\boldsymbol{\eta}^{32}=0$ as expected from the Onsager reciprocity relations (see last paragraph). Disregarding the last term in Eq.(6), the sum of (6) and (4) for $k=3$ coincides with the BPT formula, which can be written in our notations as:

$$
G^{3 j}=\frac{e}{2 \pi i} \operatorname{trace}\left(P_{\mathrm{A}} \frac{\partial S}{\partial x_{j}} S^{\dagger}\right) \quad[\mathrm{BPT}]
$$

We show later that this reduces for $j=3$ to the Landauer formula which relates the electrical conductance $G^{33}$ to the transmission of the device.

Below we explain how to derive the expressions for $\boldsymbol{\eta}^{k j}$ and $\boldsymbol{B}^{k j}$ starting from the Kubo formula Eq.(11). Later we discuss further physical implications of our results. Assuming zero temperature Fermi occupation up to energy $E_{F}$, standard textbook procedure [1, 2, 3, 4] leads to

$$
\left.\boldsymbol{\eta}^{k j}\right|_{\Gamma}=\pi \hbar \sum_{n, m} F_{n m}^{k} \overline{\delta\left(E_{F}-E_{m}\right)} F_{m n}^{j} \overline{\delta\left(E_{F}-E_{n}\right)}
$$

where the overline indicates that the delta functions are smeared. If the system were not isolated, the "broadening" $\Gamma$ of the energy levels would be determined by the interaction with the external environment [4]. But we assume a closed isolated system. Still we argue 8 that in case of a quantized chaotic system the levels acquire an effective width $\Gamma=\left(\left(\hbar \sigma_{F} / \Delta^{2}\right)|\dot{x}|\right)^{2 / 3} \Delta$, where $\Delta$ is the mean level spacing, and $\sigma_{F}$ is the root-meansquare value of the near-diagonal matrix elements (see remark [14]). Therefore we find two possibilities: In the adiabatic regime $(\Gamma \ll \Delta)$ the dissipative conductance is zero $(\boldsymbol{\eta}=0)$, while in the non-adiabatic regime $(\Gamma>\Delta)$ the dissipative conductance acquires a well defined finite value, which is not sensitive to $\Gamma$, and can be calculated using Eq.(3i). A similar claim holds regarding $\boldsymbol{B}^{k j}$, but the details are much more subtle: We start with the standard expression [5, 11]

$$
\left.\boldsymbol{B}^{k j}\right|_{\Gamma=0}=2 \hbar \sum_{n} f\left(E_{n}\right) \sum_{m(\neq n)} \frac{\operatorname{Im}\left[F_{n m}^{k} F_{m n}^{j}\right]}{\left(E_{m}-E_{n}\right)^{2}}
$$

where $f(E)$ is the Fermi occupation function (later we take the limit of zero temperature). Incorporating $\Gamma$, and exploiting the antisymmetry of the numerator with respect to $n \Leftrightarrow m$ interchange we get

$$
\left.\boldsymbol{B}^{k j}\right|_{\Gamma}=\sum_{n, m} \frac{-i \hbar F_{n m}^{k} F_{m n}^{j}}{\left(E_{m}-E_{n}\right)^{2}+(\Gamma / 2)^{2}}\left(f\left(E_{n}\right)-f\left(E_{m}\right)\right)
$$

The numerator, on the average, depends mainly on the difference $r=m-n$, and it is non-negligible within a bandwidth $\left|E_{m}-E_{n}\right|<\Delta_{b}$. We further discuss the bandwidth issue in the next paragraph, and explain that in the limit of a very long wire $\Delta \ll \Gamma \ll \Delta_{b}$. This means that in this limit $\Gamma$ serves like the infinitesimal $i 0$ in the definition of the Green functions $\mathrm{G}^{ \pm}$. Consequently, the sum in Eq.(10), which is of the form $\sum_{n, m} g(n-m)\left(f\left(E_{n}\right)-f\left(E_{m}\right)\right)=\sum_{r} r g(r)$, leads after some straightforward algebra to Eq. (15).

Formally there is an optional derivation that leads to (3) and (5). The kernel $K^{i j}(\tau)$ is related to the symmetrized correlation function $C^{i j}(\tau)=\left\langle\frac{1}{2}\left(F^{i}(\tau) F^{j}(0)+\right.\right.$ $\left.F^{j}(0) F^{i}(\tau)\right\rangle$. The quantum mechanical derivation of this subtle relation is discussed in Appendix D of [11]. If we use this relation we get from Eq.(11) an extremely simple (and useful) result:

$$
\boldsymbol{G}^{k j}=\frac{1}{\Delta} \int_{0}^{\infty} C^{k j}(\tau) d \tau
$$

which can be regarded as the generalization of the fluctuation dissipation relation. The fluctuations are described by $\tilde{C}^{k j}(\omega)$ which is defined as the Fourier transform of $C^{i j}(\tau)$. It follows from this definition that

$$
\tilde{C}^{k j}(\omega)=\left.\frac{2 \pi \hbar}{\Delta} \overline{F_{n m}^{k} F_{m n}^{j}}\right|_{E_{n}-E_{m} \approx \hbar \omega}
$$

For the device of Fig.1 the mean level spacing is $\Delta \propto 1 / L$ where $L$ is the length of the wire. The above relation implies that the bandwidth of the $m n$ matrix is $\Delta_{b} \sim \hbar / \tau_{c l}$, where the classical correlation time $\tau_{c l}$ is determined by the chaotic motion inside the dot. It is also clear that $\tilde{C}^{i j}(\omega) \propto 1 / L$, and therefore $\sigma_{F}^{2} \propto(1 / L)^{2}$. Hence we get that $\Gamma \propto(1 / L)^{1 / 3}$, implying that the limit $L \rightarrow \infty$ (keeping constant Fermi energy) is non-adiabatic, and that $\Delta \ll \Gamma \ll \Delta_{b}$. Assuming for simplicity that there is no magnetic field, one easily derives the expressions

$$
\begin{aligned}
G^{33} & =\frac{1}{2 \Delta} \tilde{C}^{33}(\omega \sim 0) \\
G^{3 j} & =\frac{1}{\Delta} \int_{-\infty}^{\infty} \Im\left[\frac{\tilde{C}^{3 j}(\omega)}{\omega}\right] \frac{d \omega}{2 \pi} \quad \text { for } j=1,2
\end{aligned}
$$

which are equivalent to those obtained in the previous paragraph. Note that $C^{3 j}(\tau)$ with $j=1,2$ is antisymmetric with respect to $\tau$, and therefore $-i \tilde{C}^{3 j}(\omega) / \omega$ is a real symmetric function.

We turn back to the formal derivation. We want to get exact expressions for the elements of the conductance matrix, for the device of Fig. 1, in the non-adiabatic limit of large $L$. The location of the particle is specified by $\boldsymbol{r}=(\mathrm{r}, s)$, where $\boldsymbol{r}$ is the coordinate along the ring, and $s$ is a transverse coordinate. Optionally we can specify the location along a lead using a radial coordinate $r$, while the surface coordinate $s$ distinguishes different points that have the same $r$. We shall refer to $r=0$ as the boundary of the scattering region. The channel basis is defined as $\langle\mathrm{r}, s \mid a, r\rangle=\chi_{a}(s) \delta\left(\mathrm{r}-\mathrm{r}_{a}(r)\right)$, where $a$ is the channel index. The wavefunction in the lead regions can 
be expanded as follows:

$$
|\Psi\rangle=\sum_{a, r}\left(C_{a,+} \mathrm{e}^{i k_{a} r}+C_{a,-} \mathrm{e}^{-i k_{a} r}\right)|a, r\rangle
$$

Following [3] we define an operator which can be identified with the imaginary part of the self energy of the interaction of the dot with the leads:

$$
\hat{\Gamma}=\sum_{a}|a, 0\rangle \hbar v_{a}\left\langle a, 0\left|=\delta(r) \otimes \sum_{a}\right| a\right\rangle \hbar v_{a}\langle a|
$$

where $v_{a}=\left(\hbar k_{a} / \mathrm{mass}\right)$ is the velocity in channel $a$. The matrix elements of the second term in Eq. (16) are

$$
\hat{\Gamma}\left(s, s^{\prime}\right)=\sum_{a} \chi_{a}(s) \hbar v_{a} \chi_{a}\left(s^{\prime}\right)
$$

Using standard procedure (see section 3.4 in [3]) the Green function in the leads, inside the scattering region $(r<0)$, can be expressed using the $S$ matrix:

$$
\begin{aligned}
& \mathrm{G}^{+}\left(r, s \mid 0, s^{\prime}\right)= \\
& -i \sum_{a, b} \chi_{b}(s) \frac{1}{\sqrt{\hbar v_{b}}}\left(\mathrm{e}^{-i k r}+S \mathrm{e}^{i k r}\right)_{b a} \frac{1}{\sqrt{\hbar v_{a}}} \chi_{a}\left(s^{\prime}\right)
\end{aligned}
$$

where $k=\operatorname{diag}\left\{k_{a}\right\}$ is a diagonal matrix. Now we are fully equipped to convert Eq. (3) into an $S$-matrix expression. Using the identities (see [3] for Eq.(19))

$$
\begin{aligned}
\operatorname{Im}\left[\mathrm{G}^{+}\right] & =-\frac{1}{2} \mathrm{G}^{+} \hat{\Gamma} \mathrm{G}^{-}=-\frac{1}{2} \mathrm{G}^{-} \hat{\Gamma} \mathrm{G}^{+} \\
\frac{\partial \mathrm{G}^{ \pm}}{\partial x_{j}} & =-\mathrm{G}^{ \pm} F^{j} \mathrm{G}^{ \pm}
\end{aligned}
$$

we obtain

$$
\boldsymbol{\eta}^{k j}=\frac{\hbar}{4 \pi} \operatorname{trace}\left[\frac{\partial \mathrm{G}^{+}}{\partial x_{j}} \hat{\Gamma} \frac{\partial \mathrm{G}^{-}}{\partial x_{j}} \hat{\Gamma}\right]
$$

Using the definition of $\hat{\Gamma}$ and Eq.(18) we get Eq.(4).

The derivation of the $S$ matrix expression Eq. (6) for $\boldsymbol{B}^{k j}$ is much more subtle, and requires a preliminary discussion of the definition of the current operator. Consider a ring geometry, and assume that the current is driven by the flux $\Phi$. In order to have a better defined model we should specify what is the vector potential $\mathcal{A}(\boldsymbol{r})$ along the ring. We can regard the values of $\mathcal{A}$ at different points in space as independent parameters (think of tight binding model). Their sum (meaning $\oint \mathcal{A}(\boldsymbol{r}) \cdot d \boldsymbol{r}$ ) should be $\Phi$. So we have to know how $\Phi$ is "distributed" along the ring. This is not just a matter of "gauge choice" because the electric field $\mathcal{E}(\boldsymbol{r})=-\dot{\mathcal{A}}(\boldsymbol{r})$ is a gauge invariant quantity. The transformation $\mathcal{A} \mapsto \mathcal{A}+\nabla \Lambda(\boldsymbol{r})$ for a time dependent filed is not merely a gauge change: A gauge transformation of time dependent field requires a compensating replacement of the scalar potential, which is not the case here. So let us define a flux $\Phi_{A}$ which is associated with a vector potential that is concentrated across a section $r=r_{A}$ of a given lead. For the later derivation it is essential to assume that the section $r=r_{A}$ is contained within the scattering region (see Fig. 1). The generalized force which is associated with $\Phi_{A}$ is $F^{3}=I_{A}$, the current through this section. Namely

$$
\begin{aligned}
I_{A}=-\frac{\partial \mathcal{H}}{\partial \Phi_{A}} & =\frac{1}{2} e\left(v \delta\left(\mathrm{r}-\mathrm{r}_{A}\right)+\delta\left(\mathrm{r}-\mathrm{r}_{A}\right) v\right) \\
& =(e / \hbar)\left[\hat{\Gamma}_{A} P^{+}-\hat{\Gamma}_{A} P^{-}\right]
\end{aligned}
$$

where $v$ is the $r$ component of the velocity operator. The last equality involves new definitions. We define

$$
\hat{\Gamma}_{A}=\sum_{a \in A}\left|a, r_{A}\right\rangle \hbar v_{a}\left\langle a, r_{A}\right|
$$

We also define projectors $P^{+}$and $P^{-}$that project out of the lead wavefunction Eq. (15) the outgoing and the ingoing parts respectively. These projectors commute with $\hat{\Gamma}_{\mathrm{A}}$. Furthermore, note that $P^{+} \mathrm{G}^{+}=\mathrm{G}^{+}$, and that $P^{-} \mathrm{G}^{+}=0$, and that $\mathrm{G}^{-} P^{-}=0$ etc. Using these extra identities one obtains the following expression:

$$
\boldsymbol{B}^{3 j}=\frac{e}{4 \pi i} \operatorname{trace}\left[\hat{\Gamma}_{\mathrm{A}} \frac{\partial \mathrm{G}^{+}}{\partial x_{j}} \hat{\Gamma} \mathrm{G}^{-}-\hat{\Gamma}_{\mathrm{A}} \frac{\partial \mathrm{G}^{-}}{\partial x_{j}} \hat{\Gamma} \mathrm{G}^{+}\right]
$$

Using the definitions of $\hat{\Gamma}$ and $\hat{\Gamma}_{\mathrm{A}}$, together with Eq.(18), followed by a straightforward algebraic manipulation, one arrives at Eq.(6) with the additional term

$$
\boldsymbol{B}_{\mathrm{intrf}}^{3 j}=\frac{e}{2 \pi} \operatorname{Re}\left[\operatorname{trace}\left(P_{\mathrm{A}} \frac{\partial S}{\partial x_{j}} \mathrm{e}^{i 2 k r_{\mathrm{A}}}\right)\right]
$$

where $P_{\mathrm{A}}$ is a projector that restrict the trace operation to the $a \in A$ lead channels.

For the simple ring geometry of Fig. 1, we have a left lead $(b \in B)$ and a right lead $(a \in A)$ channels, and the $S$-matrix can be written as

$$
S=\left(\begin{array}{cc}
\boldsymbol{r}_{\mathrm{B}} & \boldsymbol{t}_{\mathrm{AB}} \mathrm{e}^{-i \phi} \\
\boldsymbol{t}_{\mathrm{BA}} \mathrm{e}^{i \phi} & \boldsymbol{r}_{\mathrm{A}}
\end{array}\right), \quad P_{\mathrm{A}}=\left(\begin{array}{cc}
\mathbf{0} & \mathbf{0} \\
\mathbf{0} & \mathbf{1}
\end{array}\right)
$$

where $\phi=e \Phi_{\mathrm{A}} / \hbar$. Using the identity

$$
\frac{\partial S}{\partial \Phi_{\mathrm{A}}}=i \frac{e}{\hbar}\left(P_{\mathrm{A}} S-S P_{\mathrm{A}}\right)
$$

one can derive the relation that has been stated between Eq.(4), Eq.(6) and the BPT formula Eq.(7). Furthermore, assuming that there is an electro-motive force $-\dot{\Phi}_{B}$ which is induced in the other lead, one obtains from BPT $\boldsymbol{G}^{33}=\left(e^{2} / 2 \pi \hbar\right) \operatorname{trace}\left(\boldsymbol{t}_{\mathrm{AB}} \boldsymbol{t}_{\mathrm{AB}}^{\dagger}\right)$ which is the Landauer formula. The application of this procedure to multi-lead systems is a straightforward generalization.

For an open system it is evident that the current which is emitted (say) through the right lead, does not have to be equal to the current which is absorbed by the left lead. The reason is that charge can be accumulated in the dot region. But for a pumping cycle one realizes that the integrated current (pumped charge) is a well defined (lead independent) quantity. Similar observation holds in case of a closed system. Assume for example 
that the left lead is blocked. In such case raising the dot potential will cause an emission of charge through the right lead, while the current through the left lead is zero. The emitted charge is accumulated in the "wire". But for a full cycle the original charge distribution is restored, and therefore the integrated charge $(Q)$ becomes a well defined (lead independent) quantity. The additional term Eq.(26) gives a zero net contribution for a full pumping cycle. This term implies that the current is not uniform within the lead. The current has a modulation in the radial direction $(r)$, with a spatial period that equals half the De-Broglie wavelength at the Fermi energy. This reflects that the net transported current corresponds to translation of a standing wave which is associated with the last occupied level.

More subtle is the value of $Q$ for a full driving cycle. In contrast to a previous wrong statement 10] we have argued [11] that for a strictly adiabatic driving cycle, in the absence of magnetic field, the transported charge $Q$ is at best approximately quantized (say $Q \approx 1$ in units of the elementary charge). The deviation is related to the Thouless conductance of the device, and can be either positive or negative [11]. In contrast to that, with the BPT formula the correction to $Q \approx 1$ is always negative. On the basis of our derivation we can conclude the following: The deviation from quantization in a strictly adiabatic cycle is related to the contribution of the neighboring level. If the degeneracy with this level is located in the plane $\left(x_{1}, x_{2}, x_{3}=0\right)$ of the encircling cycle, then the correction is positive. If the encircled degeneracy is off plane, then the correction is negative. The effect of non-adiabaticity $(\Gamma>\Delta)$ is to screen the contribution of the neighboring levels, which is the reason for having always a negative correction from the BPT formula.

The role that dissipation may have in pumping is restricted, merely by the realization that the BPT formula is related to the Kubo formula. The Onsager reciprocity relation imply that in the absence of magnetic field the conductance matrix $\boldsymbol{G}^{k j}$ should be symmetric (antisymmetric) with respect to the permutation of the indexes $(k, j)$, depending on whether $F^{k}$ and $F^{j}$ transform (not) in the same way under time reversal. This means that shape deformations lead to dissipation via $\boldsymbol{\eta}^{i j}$ with $i, j<3$, while the electrical current is determined exclusively by the non-dissipative terms $\boldsymbol{B}^{31}$ and $\boldsymbol{B}^{32}$. This should be contrasted with the response to electro-motive force which is purely dissipative: Both the current and the dissipation are exclusively determined by the Ohmic conductance $\boldsymbol{\eta}^{33}$. Thus, in the absence of magnetic field, we have a clear cut distinction between the dissipative and the non-dissipative contributions to the response.

In summary, starting with the Kubo formalism, we were able to find expressions for the dissipative and for the non-dissipative parts of the response, and to illuminate the role of non-adiabaticity in the limiting case of an infinite system. In contradiction with past speculations, we were able to demonstrate that the switch to an open system does not necessitate an extra dissipative term.

It is my pleasure to thank T. Dittrich (Colombia), T. Kottos (Gottingen), and H. Schantz (Gottingen) for useful discussions. This research was supported by the Israel Science Foundation (grant No.11/02), and by a grant from the GIF, the German-Israeli Foundation for Scientific Research and Development.
[1] L.D. Landau and E.M. Lifshitz, Statistical physics, (Buttrworth Heinemann 2000).

[2] Y. Imry, Introduction to Mesoscopic Physics (Oxford Univ. Press 1997), and references therein.

[3] S. Datta, Electronic Transport in Mesoscopic Systems (Cambridge University Press 1995).

[4] Y. Imry and N.S. Shiren, Phys. Rev. B 33, 7992 (1986).

[5] J.M. Robbins and M.V. Berry, J. Phys. A 25, L961 (1992). M.V. Berry and J.M. Robbins, Proc. R. Soc. Lond. A 442, 659 (1993). M.V. Berry and E.C. Sinclair, J. Phys. A 30, 2853 (1997).

[6] D. J. Thouless, Phys. Rev. B27, 6083 (1983).

[7] J. E. Avron and L. Sadun, Phys. Rev. Lett. 62, 3082 (1989). J.E. Avron, A. Raveh, and B. Zur Rev. Mod. Phys. 60, 873 (1988).

[8] D. Cohen, Phys. Rev. B 68, 155303 (2003).

[9] M. Büttiker et al, Z. Phys. B94, 133 (1994). P. W. Brouwer, Phys. Rev. B58, R10135 (1998). J. E. Avron et al, Phys. Rev. B 62, R10 618 (2000).

[10] T. A. Shutenko, I. L. Aleiner and B. L. Altshuler, Phys. Rev. B61, 10366 (2000).

[11] D. Cohen, cond-mat/0208233

[12] M. Moskalets and M. Buttiker, cond-mat/0307675

[13] D.S. Fisher and P.A. Lee, Phys. Rev. B 23, 6851 (1981).
H.U. Baranger and A.D. Stone, Phys. Rev. B 40, 8169 (1989).

[14] Any segment of a pumping cycle in the $\left(x_{1}, x_{2}\right)$ plane can be regarded as "one parameter driving" with $x$ a linear combination of $x_{1}, x_{2}$, while $F$ is its conjugate.

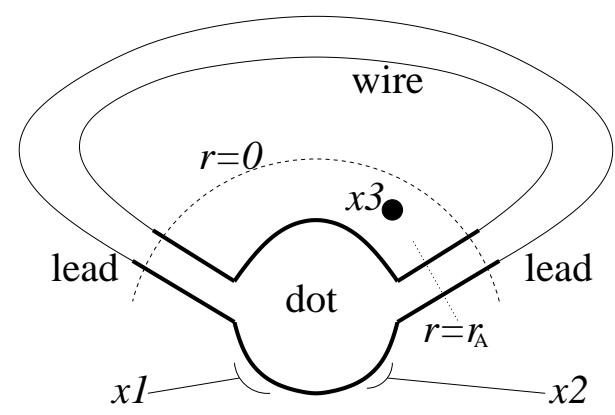

FIG1. Illustration of a closed system. The dot potential is controlled by gate voltages $x_{1}$ and $x_{2}$. The flux through the loop is $x_{3}=\Phi$. The scattering region $(r<0)$ is represented by an $S$ matrix. The length $(L)$ of the wire is assumed to be very large. 


\section{NOT PART OF THE PAPER}

\section{Going from Eq.(10) to Eq.(5)}

In this appendix we give the "straightforward algebra" that leads from Eq.(10) to Eq.(5).

$$
\boldsymbol{B}^{k j}=-i \hbar \sum_{n, m} \frac{F_{n m}^{k} F_{m n}^{j}}{\left(E_{m}-E_{n}\right)^{2}+(\Gamma / 2)^{2}}\left(f\left(E_{n}\right)-f\left(E_{m}\right)\right)
$$

Within the framework of the approximation which is discussed in the text this sum is of the form

$$
\boldsymbol{B}^{k j}=\sum_{n, m} g(n-m) \times\left(f\left(E_{n}\right)-f\left(E_{m}\right)\right)
$$

Using the notation $r=m-n$ it leads to

$$
\boldsymbol{B}^{k j}=\sum_{r} g(r) \times r
$$

We make the substitutions

$$
\begin{aligned}
r & =\frac{E_{m}-E_{n}}{\Delta} \\
g(r) & =\frac{-i \hbar F_{n m}^{k} F_{m n}^{j}}{\left(E_{m}-E_{n}\right)^{2}+(\Gamma / 2)^{2}}
\end{aligned}
$$

where $n$ is the index of an arbitrary energy level in the vicinity of the Fermi energy $\left(E_{n} \sim E_{F}\right)$.

Now we have the expression

$$
\boldsymbol{B}^{k j}=-i \hbar \frac{1}{\Delta} \sum_{m} F_{n m}^{k} \frac{E_{m}-E_{n}}{\left(E_{m}-E_{n}\right)^{2}+(\Gamma / 2)^{2}} F_{m n}^{j}
$$

Thanks to $\Gamma$ we can make the replacement $E_{n} \mapsto E_{F}$.

Next we can use (in reverse) the approximation

$$
\sum_{n}\langle n|A| n\rangle \overline{\delta\left(E_{n}-E_{F}\right)} \approx \frac{1}{\Delta}\langle n|A| n\rangle
$$

where the overline indicates a smeared delta function.

Hence we get

$$
\boldsymbol{B}^{k j}=-i \hbar \sum_{m} F_{n m}^{k} \frac{E_{m}-E_{F}}{\left(E_{m}-E_{F}\right)^{2}+(\Gamma / 2)^{2}} F_{m n}^{j} \overline{\delta\left(E_{n}-E_{F}\right)}
$$

As explained in the text, in the limit of a very long wire $\Gamma$ is like the infinitesimal $i 0$. Hence we get Eq.(5). 


\section{APPENDIX D OF REF.[8]}

\section{Expressing $\tilde{K}(\omega)$ using $\tilde{C}(\omega)$}

We can use the following manipulation in order to relate $\tilde{K}^{i j}(\omega)$ to $\tilde{C}^{i j}(\omega)$,

$$
\begin{aligned}
\tilde{K}^{i j}(\omega) & =\sum_{n} f\left(E_{n}\right) \tilde{K}_{n}^{i j}(\omega) \\
& =\frac{i}{\hbar} 2 \pi \sum_{n m} f\left(E_{n}\right)\left(F_{n m}^{i} F_{m n}^{j} \delta\left(\omega+\omega_{n m}\right)-F_{n m}^{j} F_{m n}^{i} \delta\left(\omega-\omega_{n m}\right)\right) \\
& =\frac{i}{\hbar} 2 \pi \sum_{n m} f\left(E_{m}\right)\left(-F_{n m}^{i} F_{m n}^{j} \delta\left(\omega+\omega_{n m}\right)+F_{n m}^{j} F_{m n}^{i} \delta\left(\omega-\omega_{n m}\right)\right) \\
& =\frac{i}{\hbar} 2 \pi \sum_{n m} \frac{f\left(E_{n}\right)-f\left(E_{m}\right)}{2}\left(F_{n m}^{i} F_{m n}^{j} \delta\left(\omega+\omega_{n m}\right)-F_{n m}^{j} F_{m n}^{i} \delta\left(\omega-\omega_{n m}\right)\right) \\
& =-i \omega \pi \sum_{n m} \frac{f\left(E_{n}\right)-f\left(E_{m}\right)}{E_{n}-E_{m}}\left(F_{n m}^{i} F_{m n}^{j} \delta\left(\omega+\omega_{n m}\right)+F_{n m}^{j} F_{m n}^{i} \delta\left(\omega-\omega_{n m}\right)\right) \\
& =-i \omega \sum_{n} f^{\prime}\left(E_{n}\right) C_{n}^{i j}(\omega)
\end{aligned}
$$

where we use the notation $\omega_{n m}=\left(E_{n}-E_{m}\right) / \hbar$. The third line differs from the second line by permutation of the dummy summation indexes, while the fourth line is the sum of the second and the third lines divided by 2 . In the last equality we assume small $\omega$. If the levels are very dense, then we can replace the summation by integration, leading to the relation:

$$
\tilde{K}^{i j}(\omega) \equiv \int g(E) d E f(E) \tilde{K}_{E}^{i j}(\omega)=-i \omega \int g(E) d E f^{\prime}(E) \tilde{C}_{E}^{i j}(\omega)
$$

where $\tilde{K}_{E}^{i j}(\omega)$ and $\tilde{C}_{E}^{i j}(\omega)$ are microcanonically smoothed functions. Since this equality hold for any smooth $f(E)$, it follows that the following relation holds (in the limit $\omega \rightarrow 0$ ):

$$
\tilde{K}_{E}^{i j}(\omega)=i \omega \frac{1}{g(E)} \frac{d}{d E}\left[g(E) C_{E}^{i j}(\omega)\right]
$$

If we do not assume small $\omega$, but instead assume canonical state, then a variation on the last steps in Eq.(29), using the fact that $\left(f\left(E_{n}\right)-f\left(E_{m}\right)\right) /\left(f\left(E_{n}\right)+f\left(E_{m}\right)\right)=\tanh \left(\left(E_{n}-E_{m}\right) /(2 T)\right)$ is an odd function, leads to the relation

$$
\tilde{K}_{T}^{i j}(\omega)=i \omega \times \frac{1}{\hbar \omega} \tanh \left(\frac{\hbar \omega}{2 T}\right) C_{T}^{i j}(\omega)
$$

Upon substitution of the above expressions in the Kubo formula for $\boldsymbol{G}^{i j}$, one obtains the Fluctuation-Dissipation relation.

Note added: For a low temperature Fermi occupation Eq.(30) can be written as

$$
\tilde{K}^{i j}(\omega)=i \omega g\left(E_{F}\right) \tilde{C}_{E_{F}}^{i j}(\omega)
$$

Formally this is valid only if $\hbar \omega \ll T$. But in fact if $T \ll E_{F}$ the result should not be sensitive to $E_{F}$. Therefore it can be argued that this relation holds globally, and we can set $T=0$. It follows that

$$
K^{i j}(\tau)=-\frac{\partial}{\partial \tau} g\left(E_{F}\right) \tilde{C}_{E_{F}}^{i j}(\tau)
$$

This can be used in order to derive Eq.(11) for Eq.(1). We see that it is a subtle relation which should not be regarded as a trivial identity. 